\title{
Alberto Maffi
}

\section{Gesetzgebung und soziale Ordnung in Platons Nomoi}

\author{
I.
}

Der letzte große unvollendete Dialog Platons, die Nomoi, enthalten den Entwurf einer ideellen Gesetzgebung für eine Kolonie, die auf Kreta gegründet werden sollte.

Der Gesetzgebung, die in den Nomoi vertreten wird, liegt eine besondere Absicht zugrunde. Erziehen hieß für Platon, das Verhalten der Bürger möglichst bis in die letzten Einzelheiten zu regeln. Um dieses Ziel zu erreichen, führte Platon im Rahmen der Gesetzgebung ein neues Mittel ein: die sogenannten Prooimia. Das Prooimion sollte jedem Gesetz als Mahnung vorangehen. Wenn die Bürger der Mahnung nicht gehorchten, dann sollte das Gesetz diesen Ungehorsam bestrafen.

Der Mechanismus Gehorsam / Ungehorsam war aber nicht so einfach anwendbar: Denn in vielen Fällen, besonders wenn es sich um schwere Verbrechen handelte, mußte erst entschieden werden, ob der Verbrecher heilbar war oder nicht. Die Metapher "heilbar", die aus der Sprache der Medizin kommt, war deshalb angebracht, weil Platon den Verbrecher in gewisser Weise als Kranken betrachtete. Platon hielt nicht viel von der menschlichen Natur und glaubte, sie widerstehe dem Gesetzgeber und sei imstande, alle Erziehungsergebnisse zunichte zu machen. (Im allgemeinen ist die Entscheidung den Richtern anvertraut: 957 e; im Falle des Diebstahls öffentlicher Güter ist aber die Entscheidung automatisch: Der Bürger wird zum Tode verurteilt.)

Platon stand vor einem Paradox: Eigentlich hätte die durch die Gesetzgebung erzielte Erziehung Gesetze überflüssig machen müssen, da aber im Vorgang des Erziehens auch das Scheitern mit inbegriffen sein konnte, sollten die Gesetze auch Verbrechen und Strafen berücksichtigen, die seit vielen Jahrzehnten ein beliebtes Thema der Forschung sind. Strafen war aber nur ein Teil jenes Mechanismus von sozialen Kontrollen, der sich in seiner Gesamtheit viel komplizierter und nuancierter darstellte. Nur durch diesen Mechanismus glaubte Platon die soziale Ordnung gesichert, und nur dadurch schien ihm die Gesetzgebung ihre Ziele erreichen zu können. 
Manche Aspekte sind in der Tat von Platon im Ungewissen gelassen worden: Einzelheiten, die noch nicht vorgesehen waren, sollten von jüngeren Gesetzgebern geregelt und den Grundlinien des großen gesetzgeberischen Entwurfs angepaßt werden. Aber trotz dieses Mangels sind die Grundprinzipien der Gesetzgebung Platons ziemlich deutlich entwickelt.

Die Wirksamkeit der Gesetze wird von Platon nicht nur den Magistraten, sondern auch den einfachen Bürgern anvertraut. Behörden und Bürger sind also gemeinsam verantwortlich für die korrekte Anwendung der Gesetze: Wie Platon schreibt, sollen die Bürger zusammen mit den Behörden die Verbrecher bestrafen (sunkolazein: V $730 \mathrm{~d}$ ).

Kaum eine Rolle spielten dagegen die Bürger bei der Einführung neuer Gesetze oder bei der Änderung der bestehenden Gesetze. Die Durchsetzung von neuen Gesetzen war in der Tat unentbehrlich, nicht nur um den gesetzgeberischen Rahmen zu vollenden, sondern auch, weil Platon ein grundsätzliches Muster (paradeigma) aufzubauen gedachte, dessen Realisierbarkeit jedoch im Einzelfall zu prüfen war: Die Gesetze, die sich als nicht anwendbar erweisen würden, sollten getilgt oder geändert werden.

Bei dieser Anpassung der Gesetze an die Realität spielten die Bürger keine aktive Rolle. Das heißt: Auch wenn eine Volksversammlung einberufen wurde, um neue Gesetze zu bestätigen, besaß kein Bürger ein Antragsrecht. Dieser Tatbestand läßt sich ableiten aus dem Abschnitt über den Bürger, der aus dem Ausland zurückkehrt: Ihm wird besonders streng verboten, die bestehenden Gesetze zu kritisieren; dies schließt meines Erachtens auch irgendwelche Antragsbefugnis gegenüber der Volksversammlung aus.

Diese grundsätzliche Entpolitisierung der Bürger (darauf werden wir später noch zurückkommen) steht in scheinbar paradoxem Gegensatz zum Prinzip der Rechenschaftspflichtigkeit aller Amtsträger (upeuthynoi). Denn jeder Bürger darf eine Klage gegen die Behörden anstrengen. Dies ist ein Paradox, denn gerade dieses Prinzip kennzeichnet die Demokratie schon bei Herodot: Ich verweise auf die persische Verfassungsdebatte in III 80. In der Tat beruht die Rechenschaftspflicht der Amtsträger in den Nomoi auf einer anderen Grundlage. Die archai (Amtsträger) werden in den Nomoi mehrmals als „Sklaven der Gesetze“ bezeichnet. Die Amtsträger sind also rechenschaftspflichtig nicht, weil die Volkssouveränität dies vorsehe, sondern um die korrekte Anwendung der Gesetze ihrerseits zu sichern.

Die Rechenschaftspflicht wird von Platon sogar in einer besonders strengen Art betont. Nicht nur gegen die Magistrate, sondern auch gegen Richter kann Anklage erhoben werden. Außerdem dürfen die Euthynoi selbst, das heißt die Magistrate, die für die Rechenschaft zuständig sind, von beliebigen Bürgern angeklagt werden. 
II.

Ich komme nun zu den Mitteln und Wegen, die die Wirksamkeit der Gesetze sichern sollen. Der Klarheit wegen werde ich diese Mittel in drei Kategorien einteilen: in soziale, verwaltungsrechtliche und prozeßrechtliche.

Als soziale Mittel können wir auf der einen Seite Lob und Tadel betrachten, auf der anderen Seite das unmittelbare Eingreifen einzelner Bürger.

Was Lob und Tadel betrifft, so gilt es zu betonen, daß dieses Mittel, das wir schon seit Homer als spontanes, ich würde sagen, naives Instrument der gesellschaftlichen Bewertung kennen, unter die Kontrolle des Gesetzgebers gestellt wird.

Wenn man diejenigen Fälle betrachtet, die zu dieser Form von Sanktion oder von Reaktion Anlaß geben, hat man den Eindruck, daß Platon sie benutzt, wenn er sich herkömmlicher Werte (mit Dover würde ich von Volksethik sprechen) entgegensetzt. Unterlassungstatbestände fallen einem dabei ein: Zum Beispiel werden die Bürger bestraft, die ihrer Anzeigepflicht nicht nachkommen. Im allgemeinen werden die Bürger aufgefordert, sich in die Angelegenheiten der Mitbürger einzumischen (ich verweise z. B. auf jenen Freien, der ein Sklavenkind oder dessen Pädagogen oder Lehrer nicht oder nicht richtig bestraft, obgleich dies nötig wäre: $808 \mathrm{e})$.

Gegen traditionelle Werte richtet sich auch jene Norm, die öffentliches Lob für Verwandte vorsieht, die eine vom Gesetz festgelegte Summe für die Ausrichtung von Hochzeitsfeiern nicht überziehen. Hier wird die Norm sogar dadurch verstärkt, daß Platon bei Zuwiderhandlung eine Buße vorschlägt.

Bis zum Extrem wird diese Tendenz getrieben mit der Norm, die jenen Dichtern alle Reputation abspricht, die ihre Werke auch dann unters Volk bringen, wenn der Leiter des Erziehungswesens diese bereits abgelehnt hat. Wir wissen, daß Platon von den Dichtern verlangte, daß sie agathoi kai timioi en te polei seien, auch wenn sie künstlerisch weniger begabt waren (829 d). Es mutete in der Tat als ein Paradox an, daß Dichter, die vom Gesetzgeber als die besten anerkannt werden, unter Umständen einen schlechten Ruf haben.

Wie die öffentliche Meinung beeinflußt wird, sagt Platon in den meisten Fällen nicht.

Ein besonders strenges Mittel war der Fluch. Aber Platon wagte kaum, dieses Mittel, außer bei Fällen von herkömmlichen Gesetzesmißbräuchen, in Anwendung zu bringen. Von Vergehen wie Mord oder Beleidigung der Eltern abgesehen, wird der Fluch nur gegen solche Bürger als angemessen betrachtet, die Geld aus wertvollem Metall besaßen, und solche, die dies nicht zur Anzeige brachten (742 b). Wir können vermuten, daß eine solche Ara von einem Amtsträger ausgesprochen werden sollte, wie es manche Inschriften vorschreiben (z.B. in den Dirae Teiae).

In anderen wenigen Fällen sollte ein derartiges Verbrechen auf der Agora schriftlich publik gemacht werden, so daß das Schrifttum auf unbestimmte Zeit auf die Schande hinwies (755 a) (s. unten). 
In den meisten Fällen wird aber die Art der Orientierung nicht festgestellt. Platon sagt nur, daß Lob und Tadel gemäß Gesetz (kata nomon) ausgesprochen werden sollten $(774 \mathrm{c})$. Man hat dennoch den Eindruck, daß Platon an einer Kontrolle aller Mündlichkeit und Kommunikation gelegen war, denn in der griechischen Polis blieb die öffentliche Meinung von der Mündlichkeit geprägt (957 c-d).

\section{III.}

Betrachten wir jetzt die anderen Mittel, die die Wirksamkeit der Gesetze sichern sollten. In der Anwendung der verwaltungsrechtlichen und der prozeßrechtlichen Mittel spielten die Magistrate die wichtigste Rolle. Um die Aufrechterhaltung des Staates zu sichern, durften die Magistrate einem Freien drohen und ihn tadeln (wie wir 879 e lesen), sie durften auch Bußen verhängen und Recht sprechen: Für die Rechtsprechung der Magistrate wurde eine Wertgrenze festgelegt; über diese Grenze hinaus waren entweder andere Magistrate oder besondere Gremien zuständig.

Außerdem durften und sollten die Magistrate eine Klage anstrengen: epagein diken tini, $881 \mathrm{e}$, oder gewöhnlich diken eisagein eis to dikasterion; $928 \mathrm{~b}$ eisagein eis to dikasterion bezeichnet sowohl die Klage des Vormunds gegen den Magistrat als auch die Klage der Verwandten des Mündels oder irgendeines Bürgers gegen den Vormund; $928 \mathrm{c}$ findet man diken lachein.

Obwohl nach 735 a die Befugnisse der Magistrate von den Gesetzen festgelegt werden sollten (ein eidos tes politeias ist nomous apodounai tais archais), sollten die Magistrate ihre Befugnisse offenbar ohne besondere Reglementierung ausüben dürfen: Als Gegenteil war, wie wir gesehen haben, die Rechenschaftspflicht vorgesehen.

So läßt sich verallgemeinernd sagen, daß die Magistrate von Magnesia mehr Macht gehabt hätten als die athenischen Behörden; sie hätten sich vielleicht den Magistraten der oligarchischen Poleis angenähert.

Das Gegengewicht zu dieser Art von Machtfülle bildete das System der Kontrollen. Die Magistrate waren nicht nur den Euthynoi rechenschaftspflichtig; sie sollten auch von jedem Einzelbürger gerichtlich belangt werden dürfen. Wir haben schon gesehen, daß sowohl Magistrate als auch Richter im Falle von Ungerechtigkeiten belangt werden durften. Die wichtigste Stelle hierzu ist 846 b 4-6: ean de tis tôn archontôn dokêi met' adikou gnômês krinein tas zêmias, tôn diplasiôn bupodikos estô tôi blaphthenti: ta de au tôn archontôn adikêmata eis ta koina dikastêria epanagein ton boulomenon bekastôn tôn enklêmatôn.

"If any of the magistrates be thought to have given an unjust verdict in deciding the penalties, he shall be liable to pay to injured party double the amount; and who so wishes shall bring up the wrong-doings of the magistrates before the public courts in the case of each complaint." (Bury) 
Die Worte krinein tas zemias können hier in dem Sinn verstanden werden, daß sowohl gegen die Bußen als auch gegen die eigentlichen Urteile Berufung eingelegt werden durfte.

Gegen Adikemata der Magistrate sollte jeder Bürger zu jedem Zeitpunkt eine Klage anstrengen dürfen: Es wurde nicht zwischen Privatklage und öffentlicher Klage unterschieden. Wir wissen andererseits, daß sowohl in Athen (AP 45) als anscheinend auch in Gortyn (IC IV 72, I 54-56) Privatklagen erst nach den jeweiligen Amtsperioden angestrengt werden durften.

Was die Kontrolle über die Richter betraf, so ist 767 e die wichtigste Stelle:

ean de tis epaitiatai tina hekonta adikôs krinai tên dikên, eis tous nomophulakas iôn katêgoreitô: bo de ophlôn tên toiautên dikên hupechetô men tou blabous tôi blaphthenti to hêmisu tinein, ean de meizonos axios einai doxêi zêmias, prostiman tous krinantas tên dikên hoti chrê pros toutôi pathein auton ê apotinein tôi koinôi kai tôi tên dikên dikasamenôi.

"Anyone who accuses a judge of deliberately giving an unjust judgment shall go to the Law-wardens and lay his charge before them: a judge that is convicted on such a charge shall submit to pay double the amount of the damage done to the injured party; and if he be held to deserve a greater penalty, the judges of the case shall estimate what additional punishment must be inflicted, or what payment made to the State and to the person who took proceedings." (Bury)

(Nach Ritter ${ }^{1}$ soll hier to diplasion eingesetzt werden, im Vergleich zu $762 \mathrm{~b}$ und 846 b. Aber von Einzelrichtern ist in den Nomoi nirgendwo die Rede. Es wird also verständlich, daß ein jeder nur die Hälfte des Schadens wiedergutmachen sollte. Auf jeden Fall läßt diese Norm manche Fragen offen. Was sollte mit dem Urteil geschehen? Sollte es irgendwann ungültig werden? Oder sollte es gültig bleiben, wenn die meisten Richter ein gutes Gewissen hatten? Oder sollte das Urteil immer gültig bleiben? Und was passierte, wenn der Angeklagte zum Tode verurteilt worden war?)

\section{IV.}

Wir wollen endlich die Rolle der Bürger betrachten. Wie wir gesehen haben, waren die Bürger aufgerufen, an die Seite der Magistratur zu treten, um die Gesetze durchzusetzen.

In manchen Fällen waren die Bürger gehalten, unmittelbar einzuschreiten. Es handelte sich insbesondere um Hilfe in Notlagen ${ }^{2}$ (von apagoge hingegen spricht Platon anscheinend nur in einem Fall: 879 d5-e6: Der Fremde, der einen Bürger geschlagen hat, wird von diesem zu den Astynomoi geführt).

In anderen Fällen waren die Bürger gehalten, einen Verbrecher anzuzeigen (ein allgemeines Lob einer solchen Anzeige finden wir in $730 \mathrm{~d}$ ).

1 C. Ritter, Platos Gesetze. Commentar zum griechischen Text (Leipzig 1896).

2 W. Koch, Die Strafbestimmungen in Platons Nomoi (Wiesbaden 1960) $115 \mathrm{ff}$. 
In beiden Fällen wurde eine Unterlassung bestraft.

Ein drittes Mittel, das dem Bürger zur Verfügung stand, war die gerichtliche Klage und besonders die graphe, die Klage, die öffentliches Interesse schützen sollte.

\section{V.}

Besonders wichtig für die Charakterisierung der sozialen Ordnung von Magnesia war die Begrenzung aller Möglichkeiten der Bereicherung.

Die Bevölkerung war in vier Zensusklassen eingeteilt. Wenn der Reichtum eines Bürgers das vierfache des niedrigsten Zensusbetrages erreichte, sollte der darüberliegende Betrag an die öffentliche Kasse abgegeben werden. Wenn der Bürger es nicht tat, und wenn sein Verbrechen entdeckt wurde, wurde eben dieser Teil eingezogen (vermutlich von Amts wegen als verwaltungsrechtliche Maßnahme). Außerdem durfte jeder beliebige Bürger Klage gegen ein solche Person erheben (754 e-755 a). Die Sanktion war eine Ehrenstrafe, die mit der historischen atimia nicht völlig übereinstimmte. Dem Verurteilten ging keines seiner Rechte verloren; allerdings durfte er auch keinen Anspruch auf die Vorteile erheben, die die Polis den Bürgern eventuell in Zukunft erteilen sollte. So erfahren wir mittelbar, daß es keine mistho $i$ in Magnesia gab, sondern eine Art von Verteilung der Gewinne (und der Verluste: 744 b), die die Solidarität der Bürger offenbar stärken sollte und die an eine archaische Fassung des Stadtschatzes erinnert. Vermutlich dachte Platon an solche Konsequenzen, wenn er an anderen Stellen der Nomoi einen Bürger als kakos erklären ließ.

Zudem sollte der Name des Verurteilten lebenslang auf der Agora geschrieben stehen. Hier scheint Platon die Schrift als moderneres Medium betrachtet zu haben als den schlechten Ruf. Eine Erklärung dieser Maßnahme, die in den Nomoi selten vorkommt, mag in der Tatsache zu finden sein, daß das Urteil als Schlußakt eines formellen gerichtlichen Verfahrens eine konkrete Publikationsform verlangte. Die Schriftform erlaubte auch die Setzung eines Termins, bis zu welchem die Strafe zu gelten hatte: Eine mündliche kakos-Erklärung hätte nur dann präzise sein können, wenn ein Magistrat jedes Jahr in feierlicher Form den Urteilsspruch gegen den Verurteilten wiederholt hätte, was aber Platon nirgendwo in Verbindung mit der gerichtlichen Verurteilung vorgesehen hatte.

Phthonos und die daraus folgenden Konflikte wegen der Wiederverteilung von Reichtums sollten auf diese Weise vermieden werden. Eine Folge dieser Regeln war aber auch das Ende des Evergetismus, nämlich des wichtigsten Mechanismus zur Lösung der sozialen Konflikte innerhalb der Polis. Von Leiturgien sprach Platon beiläufig, wenn ich recht gesehen habe, nur einmal ( $949 \mathrm{~d}$ ).

Die Knappheit an Geld behinderte auch die Anleihemöglichkeiten: So wurde der Hauptgrund des Schuldproblems, das so viele griechische Poleis ängstigte, völlig getilgt. Als zusätzliche Maßnahme wurde verfügt, Kredite als nicht einklagbar zu deklarieren. 
Wir wissen nicht, was geschah, wenn ein Verurteilter nicht genug Geld hatte, um die Strafsumme zu bezahlen. In $855 \mathrm{~d}$ war das Gefängnis vorgesehen; es handelte sich aber um zemiai, die von einem Magistrat auferlegt wurden.

Aus diesem knappen Überblick mag man sehen, wie ernst Platon Probleme der sozialen Ordnung nahm: Hauptmittel zu deren Lösung war für ihn die Erweiterung der Rolle des Gesetzgebers. Heute sind alle civil-law-Systeme durch eine fast unübersehbare Menge von Gesetzen geprägt; aber mit der sozialen Ordnung hat der Gesetzgeber nichts mehr zu tun. So kann man sagen, daß das platonische Projekt sich nur zum Teil verwirklicht hat, und man kann sich die Frage stellen, ob der beste Teil davon verwirklicht worden ist. 
